\title{
KSHV Regulation of Fibulin-2 in Kaposi's Sarcoma
}

\author{
Implications for Tumorigenesis
}

\author{
Donald J. Alcendor, ${ }^{*}$ Susan Knobel, ${ }^{*}$ \\ Prashant Desai, ${ }^{\dagger}$ Wen Qui Zhu, ${ }^{*}$ and \\ Gary S. Hayward ${ }^{\dagger}$ \\ From the Meharry Medical College," School of Medicine, Center \\ for AIDS Health Disparities Research, Nashville, Tennessee; and \\ the Department of Viral Oncology and Pharmacology, ${ }^{\dagger}$ Molecular \\ Virology Laboratories, Sidney-Kimmel Cancer Research Center, \\ Johns Hopkins University School of Medicine, Baltimore, \\ Maryland
}

Kaposi's sarcoma is an angioproliferative tumor caused by Kaposi's sarcoma-associated herpesvirus (KSHV) infection of vascular endothelial cells. Fibulins, proteins that associate with extracellular matrix (ECM) proteins, may have both tumor-suppressive and oncogenic activities. We found that the expression of fibulin-2 protein and mRNA were decreased 50-fold and 26-fold, respectively, in 10-day KSHVinfected dermal microvascular endothelial cells (DMVEC). Using quantitative RT-PCR, we found a fivefold and 25-fold decrease of fibulin-2 extracellular matrix binding partners, fibronectin and tropoelastin, respectively. Time-course transcriptional analyses over 10 days showed that in addition to that of fibulin-2, expression of fibulins 3 and 5 was decreased in KSHVinfected DMVEC, fibulins 1C/1D were increased, and fibulins 4, 6, and 7 were unchanged. KSHV latencyassociated nuclear antigen (LANA) transcription levels rose consistently over the same period. Addition of recombinant fibulin-3 or -5 for 48 hours to 10-day KSHV-infected cells caused a suppression of KSHVinduced vascular endothelial growth factor (VEGF) protein and mRNA levels. Recombinant fibulin-3 also significantly reduced VEGF receptor 3 expression. In pleural effusion lymphoma cell lines that express variable levels of KSHV lytic replication, we observed no detectable fibulin- 2 or -5 expression. Finally, fibulin-2 expression was decreased in tissue microarrays from KSHV-infected, LANA-positive patient cells as compared to that in patient nontumor controls. Understanding the interactions between KSHV and the fibulins may lead to the development of novel therapies for treatment of Kaposi's sarcoma. (Am J Pathol 2011, 179:1443-1454; DOI: 10.1016/j.ajpath.2011.05.024)

Before highly active antiretroviral treatment in 1996, at the height of the HIV/AIDS epidemic, the incidence of Kaposi's sarcoma (KS) increased 20,000-fold among gay males in the United States ${ }^{1,2}$ and is now the predominant HIV/AIDSrelated malignancy in Southern Africa and, hence, the world. ${ }^{3}$ Kaposi's sarcoma-associated herpesvirus (KSHV) or Human Herpesvirus type $8(\mathrm{HHV} 8)$ is the etiological agent of $\mathrm{KS} .{ }^{4,5} \mathrm{KS}$ is characterized as an angioproliferative tumor caused by KSHV infection of vascular endothelial cells and produces rare B-cell lymphoproliferative diseases in the form of pleural effusion lymphomas (PEL) and some forms of Multicentric Castleman's Disease. KS lesions are usually papular in nature, but can be plaque-like as well, and in a more advanced stage, they develop into nodular tumors with extensive organ involvement. ${ }^{6}$

Fibulins comprise a seven-member family of extracellular matrix glycoproteins that share a carboxyl-terminal globular domain plus a series of calcium-binding epidermal growth factor (EGF)-like motifs, and are known to be highly conserved throughout evolution. ${ }^{7,8}$ The human fibulin-1 protein contains 20 exons and, by the process of alternative splicing, yields four separate transcript variants designated $1 \mathrm{~A}$ to $1 \mathrm{D} .^{7-9}$ Fibulin-2, the primary focus of this study, is the second largest member of the fibulin family because of a unique 400-amino acid N-terminal domain. ${ }^{10}$ Fibulins 3,4 , and 5 are relatively smaller in size (50 to $60 \mathrm{kDa}$ ) and share a higher degree of structural similarity to one another. ${ }^{11}$ They are characterized by a modified EGF-like motif at the N-terminus upstream of five

Supported in part by Vanderbilt-Meharry Center for AIDS Research (CFAR) [National Institutes of Health (NIH) grant P30Al054999]; the Center for AIDS Health Disparities Research (NIH grant U54RR019192); Meharry Center for Clinical Research (CRC); and NIH grant P2ORR011792. D.J.A. was funded by pilot grants from the CFAR and the Meharry CRC. Accepted for publication May 17, 2011.

Address reprint requests to Donald J. Alcendor, Ph.D., Meharry Medical College, School of Medicine, Center for AIDS Health Disparities Research, 1005 Dr. D.B. Todd Jr. Blvd., Nashville, TN 37208-3599. E-mail: dalcendor@mmc.edu. 
tandem EGF repeat elements and a fibulin-type C terminal domain. Fibulin-6 is the largest of the fibulin family members and is distinguished by its large $\mathrm{N}$-terminal immunoglobulin C-2 domain. ${ }^{12}$ Fibulin-7 is the most recently discovered member of this family and is the human homolog of the mouse fibulin-7 gene also known as TM14. ${ }^{13}$ The smallest of the fibulins, human fibulin-7 isoform-1, is only 439 amino acids and has a unique sushi domain with only three EGF-like motifs. ${ }^{13}$

Fibulins are reported to have both tumor-suppressive and oncogenic activities as indicated by the following examples: i) fibulin-1 is overexpressed in ovarian epithelial tumors and breast cancer, ${ }^{14,15}$ whereas loss of fibulin-2 expression is linked to breast cancer progression ${ }^{16}$; ii) fibulin-3 antagonizes tumor angiogenesis in vivo, and its expression is decreased in various tumors ${ }^{17}$; iii) fibulin-4 expression is increased in colon cancer ${ }^{18}$; and iv) fibulin-5 expression is increased in the HT1080 fibrosarcoma cell line and might inhibit angiogenesis. ${ }^{17,19} \mathrm{How}$ ever, fibulin-5 expression is also reported to be decreased in ovarian, colon, kidney, and breast cancer. ${ }^{19}$ Fibulin-6 and -7 have yet to be linked to cancer.

Thus fibulins are ECM proteins involved in cell adhesion, proliferation, migration, invasion, and angiogenesis that have been linked to progression of several cancer types, but have not been studied in KS. We examined fibulin-2 in studies leading up to this report because we found it to be significantly down-regulated by microarray analysis in KSHV-infected DMVEC cells. Because of our results, we also assayed other fibulins for their activities after KSHV infection of DMVEC cells, as well as examined KS archival tissue for expression of fibulin-2. Notably, we found fibulins 2, 3, and 5 to be down-regulated over time in KSHV-infected DMVEC, whereas fibulins $1 \mathrm{C}$ and $1 \mathrm{D}$ were up-regulated, with no change in fibulins 4,6 , and 7 . This represents the first study that examines fibulin-2 expression in KSHV-infected DMVEC and KS. Our results suggest that changes in expression of these ECM proteins may play a role in tumor progression.

\section{Materials and Methods}

\section{Cells and Viruses}

The BCBL-1 cell line originally isolated from a body cavitybased lymphoma was cultured in RPMI 1640 medium (Gibco, Grand Island, NY; supplemented with 10\% FBS, $10 \mathrm{mmol} / \mathrm{L}$ HEPES, L-glutamine) until the cell density reach $3 \times 10^{6}$ cells $/ \mathrm{mL}$. Lytic cycle virus replication was then induced with TPA $(20 \mathrm{ng} / \mathrm{mL})$ and sodium butyrate (0.3 ng/mL). Twenty-four hours after induction, cells were washed twice in PBS to remove butyrate, and induction was continued with TPA for 5 days. Cell-free virus was isolated and concentrated by differential centrifugation. DMVEC maintained in complete EMB-2 medium (Lonza, Basel, Switzerland) obtained from Lonza Corporation were infected at passage 4 at a multiplicity of infection of 0.01 ; mock-infected cells were used as controls. $B J A B$ (B-cell control) and PEL cell lines JSC-1, BC3, BC2, BCBL-1, and HBL6 were maintained in RPMI 1640 me- dium supplemented with $10 \%$ fetal calf serum and 100 $\mu \mathrm{g} / \mathrm{mL}$ of penicillin/streptomycin.

\section{Giemsa Staining}

Normal and KSHV-infected DMVEC cells were cultivated in chamber slides at $80 \%$ confluence. Medium was removed, and cells were washed three times with PBS $(\mathrm{pH} 7.4)$ then fixed for 10 minutes in absolute methanol at $-20^{\circ} \mathrm{C}$ for 15 minutes. Giemsa stock stain was diluted in Giemsa buffer and cells were stained according to the manufacturer's recommendations (Invitrogen, Carlsbad, CA). Stained cells were dried and sealed with a glass coverslip using permanent mounting medium. Slides were viewed on a Nikon TE2000S microscope (Nikon, Tokyo, Japan) under brightfield illumination at a total magnification of $\times 200$.

\section{Western Blot}

To determine levels of fibulin-2 protein expressed by DMVEC cells before and after KSHV infection, extracts from infected and mock-infected cells were prepared using radioimmunoprecipitation assay lysis buffer [50 $\mathrm{mmol} / \mathrm{L}$ Tris (pH 7.5), $150 \mathrm{mmol} / \mathrm{L} \mathrm{NaCl}, 2 \mathrm{mmol} / \mathrm{L}$ EDTA (pH 8.0), 1\% NP40, $0.5 \%$ deoxycholate sodium, $0.1 \%$ SDS, and $0.25 \mathrm{mg} / \mathrm{mL}$ proteinase inhibitor]. Lysates were placed on ice for 30 minutes and then clarified by centrifugation. Supernatants were removed and total protein measured by BCA assay (Pierce; Thermo Fisher Scientific, Waltham, MA). Fifteen micrograms of protein lysates for mock-infected and infected paired samples were fractionated in $4 \%$ to $20 \%$ SDS-PAGE gels, transferred to nitrocellulose membranes, blocked with $5 \%$ milk powder, $0.1 \%$ TBST $(0.1 \%$ Tween $20,20 \mathrm{mmol} / \mathrm{L}$ Tris, $150 \mathrm{mmol} / \mathrm{L}$ $\mathrm{NaCl}$ ) and incubated separately at $4^{\circ} \mathrm{C}$ overnight with a primary rabbit antibody fibulin-2, LANA, and $\beta$ actin, all at a 1:2000 dilution. Membranes were washed five times in washing buffer (0.1\% TBST) and incubated for 1 hour, followed by a secondary mouse anti-rabbit peroxidase conjugate (Jackson ImmunoResearch Laboratories, West Grove, PA), at a dilution of 1:10,000. Immunoreactive bands were detected with SuperSignal West-Dura Extended Duration Substrate (Pierce; Thermo Fisher Scientific) following exposure to X-ray film.

\section{Southern Blot Hybridization}

Cultured PEL cell lines JSC-1, BC3, BC2, BCBL-1, and HBL6, and BJAB cells were washed three times with PBS, pelleted by centrifugation, and lysed with lysis buffer ( $1 \%$ gelatin, $25 \mathrm{mmol} / \mathrm{L}$ EDTA, 1\% SDS). PEL lysates were extracted with phenol-chloroform and precipitated with $2.5 \mathrm{~mol} / \mathrm{L}$ ammonium acetate in ethanol. Ten micrograms of total genomic DNA from PEL cells were electrophoresed in $0.8 \%$ agarose gels, transferred to nylon membranes, and then hybridized to a ${ }^{32} \mathrm{P}$-labeled DNA probe representing an 800-bp Notl fragment of the terminal repeat region of the KSHV genome. Denatured DNA fragments were labeled according to the manufacturer's recommendations using a random primer DNA labeling kit 
(Invitrogen). Hybridized bands were visualized by autoradiography after exposure to X-ray film.

\section{Densitometry}

Densitometry analysis was performed on immunoblots from mock- and KSHV-infected DMVEC cells using a Bio-Rad Chemi-Doc XRS gel docking system and Quantity One 4.6.2 software (Bio-Rad Laboratories, Hercules, CA).

\section{Immunohistochemistry}

Dual-labeled immunohistochemistry for fibulin-2 and KSHV LANA was performed on nodular archival AIDS-KS tissue fixed in formalin, embedded in paraffin, sectioned, and then placed on chemate slides before immunohistochemistry. For staining, antigen retrieval was performed with microwave oven pretreatment (500 W) in citrate buffer at $95^{\circ} \mathrm{C}$ for 15 minutes. Cells were then incubated 30 minutes in PBS ( $\mathrm{pH}$ 7.2) containing 10\% normal goat serum with $1 \%$ Tween-20 for blocking of nonspecific binding and permeabilization. Cells were then incubated with a rabbit polyclonal antibody to fibulin-2 (Santa Cruz Biotechnology, Santa Cruz, CA) diluted 1:100 in blocking buffer. After 90 minutes of incubation, cells were subsequently washed, and incubated with a biotinylated donkey anti-rabbit IgG antibody (Dako, Glostrup, Denmark) at a 1:100 dilution and then with a horseradish peroxidase-avidin conjugate (Dako) at 1:500 dilution. Color development was achieved by incubating cells with the substrate 3,3-diaminobenzidine (DAB, Sigma, St. Louis, $\mathrm{MO})$. The second labeling was performed by an additional antigen retrieval step in the microwave as described above and a second round of staining with a monoclonal antibody to the KSHV LANA (Vector Laboratories, Burlingame, CA) at a 1:100 dilution. Cells were subsequently washed, incubated with a biotinylated goat anti-mouse/rabbit IgG antibody (Dako) at a 1:100 dilution, and then an alkaline phosphatase-strepavidin conjugate (Vector Laboratories) at a 1:500 dilution. Color development was generated by incubating cells with the substrate Vector Red using a Vector Red Alkaline Phosphatase Substrate Kit (Vector Laboratories) according to the manufacturer's instructions. Images were taken with a Nikon TE-2000S microscope mounted with a charge-coupled device camera.

\section{Microarray Analysis}

The GeneChip Human Genome U133 Plus 2.0 array (Affymetrix, Santa Clara, CA) with complete coverage of the human genome containing over 47,000 transcripts was used to identify gene expression changes in primary DMVEC cells 10 days after KSHV infection. Several genes were found to be down-regulated after KSHV infection. Fibulin-2 was identified as being significantly down-regulated and therefore was the first fibulin selected for further study.

\section{RNA and cDNA Amplification}

Total RNA was extracted from KSHV-infected DMVEC cells and mock-infected cells using a Qiagen mini RNA isolation kit (Qiagen, Valencia, CA). The RNA was DNAase treated before elution on the column according to the manufacturer's recommendations. mRNA in $1 \mu \mathrm{g}$ of each sample was primed using oligo-dT and reverse transcribed with a High Capacity cDNA Reverse Transcription kit (Applied Biosystems, Foster City, CA).

\section{Real-Time Quantitative PCR}

Primer sets for quantitative PCR (qPCR) included fibulin-3 forward 5'-TGCCATCAGACATCTTCCAG-3', reverse 5'TGCCTGTGGTTGACTCTTAGAA-3' (292 bp); fibulin-4 forward 5'-GCCCAAACCTGTGTCA-3', reverse 5'-TGATTTGCCTAATGTAAAAGTCC-3' (288 bp); fibulin-6 forward 5'-TGCTCGGTCCAAAGATTACC-3', reverse 5'TGGAGGCCTGTACTGCTTCT-3' (227 bp); and fibulin-7 forward 5'-CTCTGCCTTCCAACCTGAAG-3', reverse 5' CAGGTTATGCACAAGGATCA-3' (179 bp) were used. Primer sequences for qPCR for fibulin-2 and fibulin- 5 were identical to primers sets used in the RT-PCR reactions. GPCR primer sets for fibulin-2 binding protein partners included aggrecan forward 5'-GCCTCAGAAAAGTGCTGACC-3', reverse 5'-GTCCCTCTGTCTCCTTGCAG-3' (197 bp); fibronectin forward 5'-CCCAACTGGCATTGACTIT-3', reverse 5'-CTCGAGGTCTTCACTGAAG-3' (136 bp); fibrillin forward 5'-GTGACTGCCCACCTGATTTT-3', reverse 5'-AGCAGGAAGCTTTGGAAACA-3' (156 bp); laminin $\alpha 2$ forward 5'-AAAGTATCTGTGTCTTCAGGAGGTGC-3', reverse 5'-GCAGCCAGTGAATGTAATCACACGTC-3' (474 bp); nidogen forward 5'-ATAGAAGTGGCGAAGCTGGA-3', reverse 5'GTGCCGTCCATGTAGGAAGT-3' (161 bp); perlecan forward 5'-GGAGCCTATTTCCACGATGA-3', reverse 5'GAGGCTGATGAAGTCCTTGC-3' (180 bp); tropoelastin forward 5'-ACCTGGGACAACTGGAATCC-3', reverse 5'AAAGCAGCAGCAAAGTTCGG-3' (288 bp); and versican forward 5'-CGGGATCCGGGGTGAGAACCCTGTATCG-3', reverse 5'-ACTCTAGAGGCCACGCCTAGCTTCTGCAGC-3' (375 bp) were used.

Primer sets for amplification of VEGF 121, 165, and 189, and VEGF receptor 1 (VEGFR1), VEGF receptor 2 (VEGFR2), and VEGF receptor 3 (VEGFR3) are as follows: VEGF 121, 165, and 189 forward 5'-GAGATGAGCTTCCTACAGCAC-3', reverse 5'-TCACCGCCTCGGCTTGTCACAT-3'; VEGFR1 forward 5'-GGAACAAGGCAAGAAACCAA-3', reverse 5'-CGATGAATGCACTTTCTGGA-3'; VEGFR2 forward 5'-ATCCCTGTGGATCTGAAACG-3', reverse 5'-CCAAGAACTCCATGCCCTTA-3'; and VEGFR3 forward 5'-TGAAAGCATCTTCGACAAGG-3', reverse 5'-TTCAGCATGATGTGGCGTAT-3'.

Real-time PCR was performed in 96-well optical plates (Sorenson Bioscience, Salt Lake City, UT) with cDNA using the MyiQ Single Color Real-Time PCR Detection System (Bio-Rad Laboratories) in $25-\mu \mathrm{L}$ reaction volumes. A master mix was made according to the manufacturer's instructions using SYBR Green Supermix (BioRad Laboratories). Veriquest SYBR Green Supermix was substituted when amplifying high GC-rich cDNA tem- 
plates according to the manufacturer's instructions (Affymetrix). Forward and reverse primers were used at a concentration of $250 \mathrm{nmol} / \mathrm{L}$ per well, made in RNAase and DNAase free $\mathrm{H}_{2} \mathrm{O}$. Primer sets for fibulin-2 binding proteins included the cDNAs from mock-infected DMVEC cells and KSHV-infected DMVEC were diluted 1:3 using RNAase DNAase free $\mathrm{H}_{2} \mathrm{O} ; 3 \mu \mathrm{L}$ of this dilution was added to each well. Control wells substituted water for cDNA. The cycling sequence included $95^{\circ} \mathrm{C}$ for 3 minutes, $95^{\circ} \mathrm{C}$ for 15 seconds, $60^{\circ} \mathrm{C}$ for 1 minute, $95^{\circ} \mathrm{C}$ for 1 minute, $55^{\circ} \mathrm{C}$ for 1 minute, and $55^{\circ} \mathrm{C}$ for 30 seconds for 81 cycles total. A GAPDH primer set was included for normalization. Data analysis was done with the Bio-Rad iQ5 Optical System Software Version 2.

\section{Human Angiogenesis ELISA}

The effects of fibulins on KSHV-induced angiogensis were measured using a human angiogenesis ELISA assay kit profiling six angiogenic cytokines (Signosis, Sunnyvale, CA). Normal and KSHV-infected DMVEC cells were plated at a density of $4 \times 10^{5}$ cells in six-well dishes and grown to $90 \%$ confluence (10 days). Normal DMVEC, KSHV-infected DMVEC, and KSHV-infected DMVEC exposed to $10 \mu \mathrm{g} / \mathrm{mL}$ of purified recombinant fibulin-3 protein (Origene, Rockville, MD) or $10 \mu \mathrm{g} / \mathrm{mL}$ of purified recombinant fibulin-5 protein (Origene) were cultured in EBM-2 media (Lonza, Walkersville, MD) supplemented with $2 \%$ fetal calf serum only for an additional 48 hours. Fibulin recombinant protein concentrations needed for biological activity were estimated based on prior studies in mice angiogenic sprouting assays. ${ }^{20}$ Cell supernatants were then assayed for angiogenic cytokines and compared to mock-infected control cells and KSHV-infected DMVEC cells, according to the manufacturer's protocol. Cell monolayers were harvested, washed three times in phosphate-buffered saline ( $\mathrm{pH} 7.4)$, and total RNA extracted for real-time PCR analysis.

\section{Tissue Microarrays}

Tissue microarrays were obtained from AIDS Cancer Specimen Resource Consortium (ACSR, San Francisco, CA). All specimens were procured and used after obtaining approval by the institutional review board of Meharry Medical College. Paraffin-embedded KS tissue of 0.6- $\mathrm{mm}$ core sizes representing patch/plaque and nodular forms of KS from multiple patients were placed on slides. KS tumor tissue from the mouth, skin, tongue, soft palate, and neck masses were included on the array. These specimens included areas of surrounding tissue (controls) that were not infected as judged by the lack of a spindle shape morphology and LANA-positive staining. Only those tissues staining positive for LANA were considered KS tumor cores. All tissues were from HIV-positive patients. When we observed a $>50 \%$ reduction in fibulin-2 expression in LANA-positive spindle cell regions in 50 optical fields compared to LANA-negative regions, then in 50 optical fields that showed a $>50 \%$ reduction in fibulin-2, then that specific tumor core was scored as
Dermal Microvascular Endothelial Cells Infected with KSHV
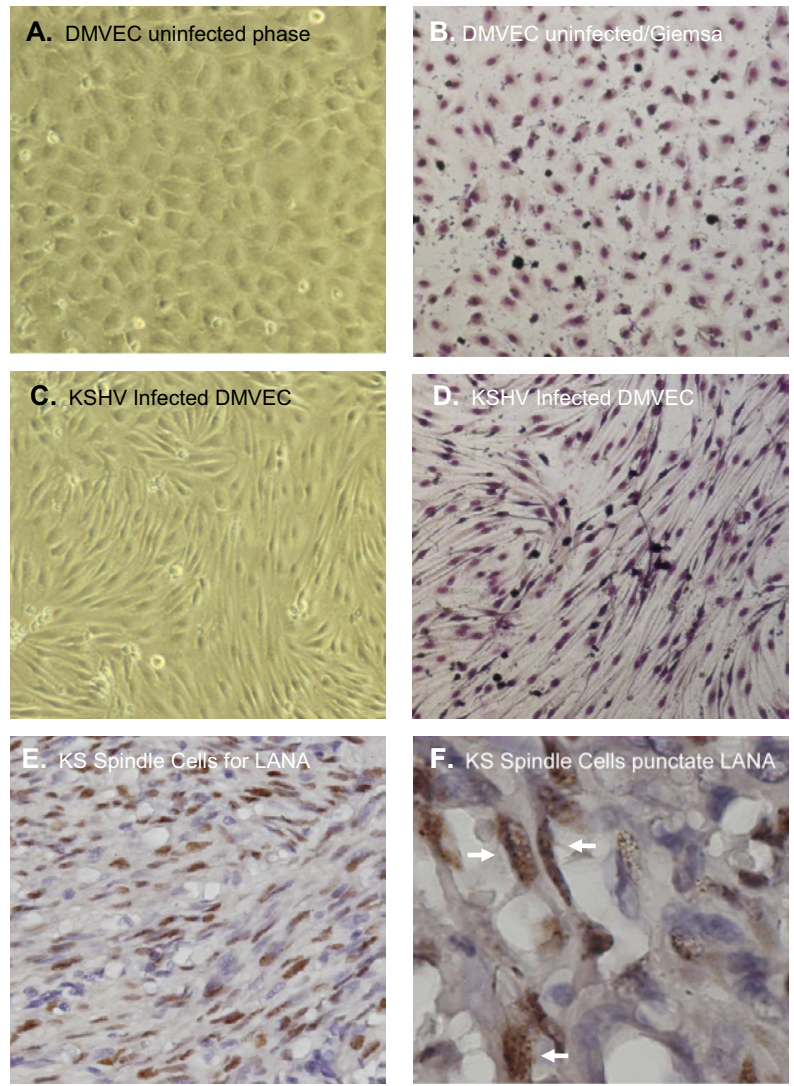

Figure 1. Human dermal microvascular endothelial cell (DMVEC) mockinfected controls and DMVEC cells infected with KSHV for 10 days. For infections, cells were treated with BCBL-1 virus at a multiplicity of infection (moi) of 0.01 and cultivated in EBM- 2 complete medium. Phase images were taken with a NIKON TE $2000 \mathrm{~S}$ microscope mounted with a CCD camera. Shown are phase $(\mathbf{A})$ and Giemsa $(\mathbf{B})$ stained images of mock-infected cells showing a cobblestone-like morphology C: KSHV-infected cells with the characteristic spindle shape morphology. D: This type of swirling/spindling appears more pronounced when a Giemsa stain is performed and appears very distinct from uninfected cells. E: An IHC stain of a nodular AIDS-KS tumor showing KSHV LANA positive spindle cells with a hematoxylin counterstain. F: Shows KS tissue with spindle cells stained positive for KSHV LANA with a nuclear punctate staining pattern (white arrows). All images were, with the exception of Figure $1 \mathrm{~F}$, taken at a total magnification of $\times 200$. The image in Figure $1 \mathrm{~F}$ was taken at a total magnification of $\times 600$ to discern the nuclear punctate staining pattern of KSHV LANA.

being reduced in expression for fibulin-2 in virus-positive cells.

\section{Results}

\section{KSHV Infection of DMVEC}

Uninfected DMVEC cells displayed a cobblestone appearance as seen by phase microscopy and Giemsa staining, which is consistent with the morphology of normal, early passaged DMVEC cells (Figures 1, A and 1B). These normal DMVEC were infected in vitro with BCBL1 virus, and 10 days after infection, distinct morphological changes were observed that were consistent with the spindle cell phenotype for KSHV-infected DMVEC (Figure $1 \mathrm{C})$ and $\mathrm{KS}$ tumors in vivo. ${ }^{6}$ This swirling cell pattern was more pronounced when the infected cells were stained 
A

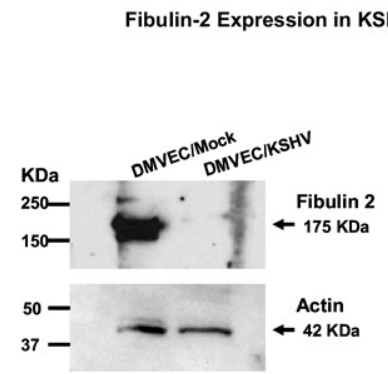

B

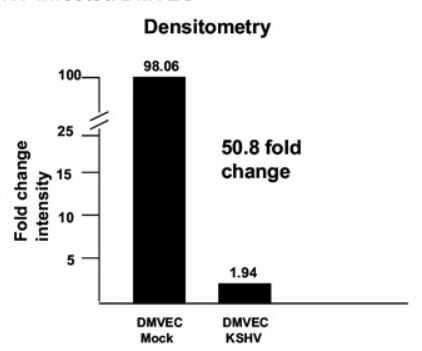

C

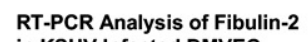
in KSHV Infected DMVEC

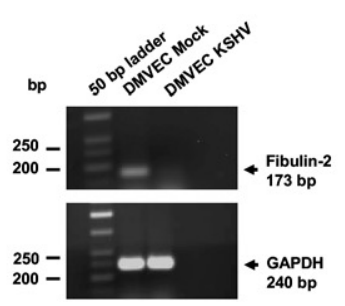

Fibulin-2 Expression by qRT-PCR in KSHV Infected DMVEC cells after 10 days

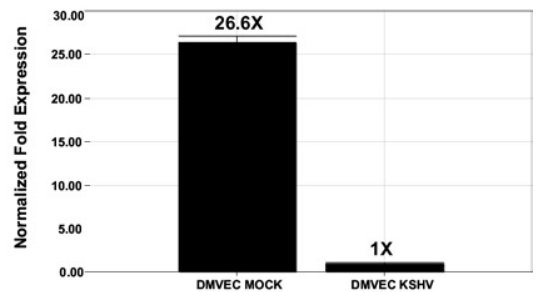

Figure 2. Analyses for fibulin-2 expression at 10 days after mock or KSHV infection of DMVEC. A: Lysates (15 $\mu \mathrm{g})$ from mock- and KSHV-infected DMVEC were separated by electrophoresis in $4 \%$ to $20 \%$ PAGE gels transferred to nitrocellulose membranes and screened for expression of fibulin-2 using a rabbit polyclonal antibody (Santa Cruz Biotechnology) at a 1:2,000 dilution. Western blot bands show evidence of a $\sim 175$ kDa protein in lysates from mock-infected DMVEC compared to the mark reduction of this band in KSHV-infected DMVEC. B: Densitometry analysis of the Western blots in Figure 2A reveals a greater than 50-fold reduction in fibulin-2 protein expression in KSHV-infected DMVEC cells at 10 days compared to mock-infected control cells. C: Semiquantitative RT-PCR analysis of fibulin-2 mRNA expression in KSHV-infected DMVEC. Ten nanograms of cDNA from mock- and KSHV-infected DMVEC were amplified by PCR using fibulin-2 gene-specific primers. PCR products were electrophoresed in 1.5\% agarose, and PCR DNA fragments were sized using a 50-bp ladder. The expected fragment size for fibulin-2 is 173 bp. GAPDH was amplified as a loading control with a fragment size of 240 bp. D: Real-time RT-PCR analysis of fibulin-2 expression in KSHV-infected DMVEC cells 10 days after infection compared to mock-infected control cells. Relative fold expression was normalized to GAPDH.

with Giemsa stain (Figure 1D). We observed a similar spindle cell morphology in an AIDS-KS tumor specimen stained positive (brown) for KSHV LANA (Figure 1E), which also showed a characteristic nuclear punctate staining pattern as shown by immunohistochemical staining of the tissue (Figure 1F).

\section{KSHV Decreases Fibulin-2 Protein and RNA Expression in DMVEC Cells}

Using microarray analysis, we observed that fibulin-2 was significantly down-regulated in KSHV-infected DMVEC cells in comparison to mock-infected control cells (data not shown). To validate our microarray data, we determined the protein expression levels of fibulin-2 in KSHVinfected DMVEC by Western blot analysis and found a significant reduction in fibulin-2 protein (Figure 2A). By densitometry analysis of the Western blot, it was determined that there was a more than 50 -fold reduction in fibulin-2 protein expression (Figure 2B).

To determine whether KSHV infection induced transcriptional down-regulation of fibulin-2 as well, we prepared cDNA from mock- and KSHV-infected DMVEC cells and performed semiquantitative RT-PCR (sqRTPCR). We show a significant down-regulation of fibulin-2 in KSHV-infected DMVEC by sqRT-PCR 10 days after infection (Figure 2C). After quantitation by real-time RTPCR (qRT-PCR), we observed a 26-fold reduction in fibulin-2 message in KSHV-infected DMVEC cells 10 days after infection compared to mock-infected controls (Figure 2D). Thus, transcriptional down-regulation of fibulin-2 correlated with the decrease seen in fibulin-2 protein expression observed by immunoblot analysis.

\section{Dysregulation of Fibulin-2 Binding Partners Fibronectin and Tropoelastin in KSHV-Infected DMVEC Cells}

After observing a 50-fold reduction in fibulin-2 expression in 10-day KSHV-infected DMVEC cells, we wanted to deter- mine the effects of fibulin-2 dysregulation on its extracellular matrix binding partners aggrecan, fibronectin, fibrillin, laminin $\alpha 2$, nidogen, perlecan, tropoelastin, and versican. ${ }^{21-26}$ Using quantitative PCR (qRT-PCR), we observed a fivefold and 25-fold down-regulation of fibronectin and tropoelastin, respectively, with no significant changes in the other fibulin-2 ECM binding partners examined (Figure 3).

\section{Transcription of Other Fibulins in KSHV-Infected DMVEC by $q R T-P C R$}

Because other fibulins have been implicated in cancer and may serve an important role in the establishment and maintenance of the extracellular matrix in these cells, we examined the transcription profile of the seven additional fibulins in 10-day KSHV-infected DMVEC cells. The results are shown in Figure 4. Within this group, we found no significant changes in mRNA expression for fibulins 4,6 ,

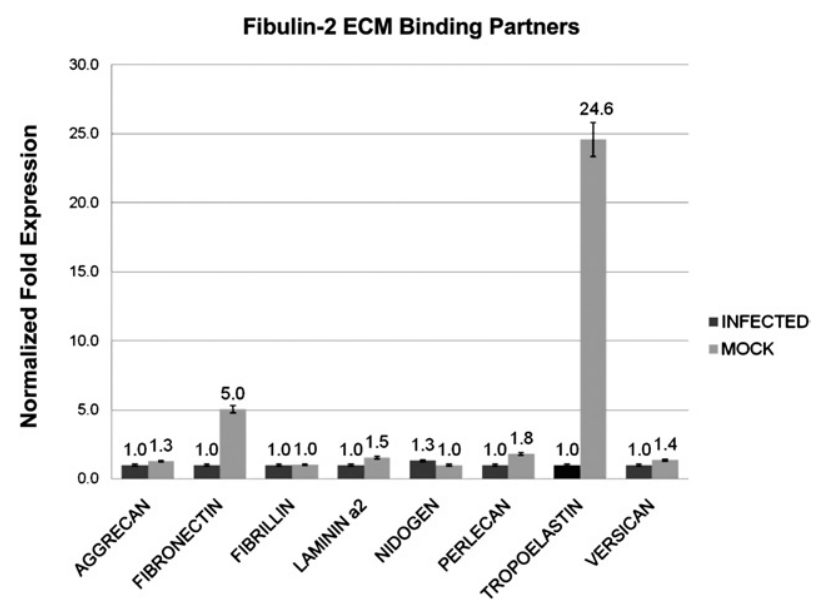

Figure 3. Real-time PCR analysis of fibulin-2 binding partners in KSHVinfected DMVEC cells. cDNA was prepared from total RNA isolated from 10-day KSHV-infected DMVEC cells along with mock-infected control cells. Black bars indicated fold transcription for KSHV-infected DMVEC cells and gray bars represent fold change in transcription of mock-infected cells. This experiment was replicated three times, and amplifications were performed in triplicate and normalized to GAPDH. 


\section{Expression of Fibulins by qRT-PCR in KSHV Infected DMVEC Cells after 10 days}
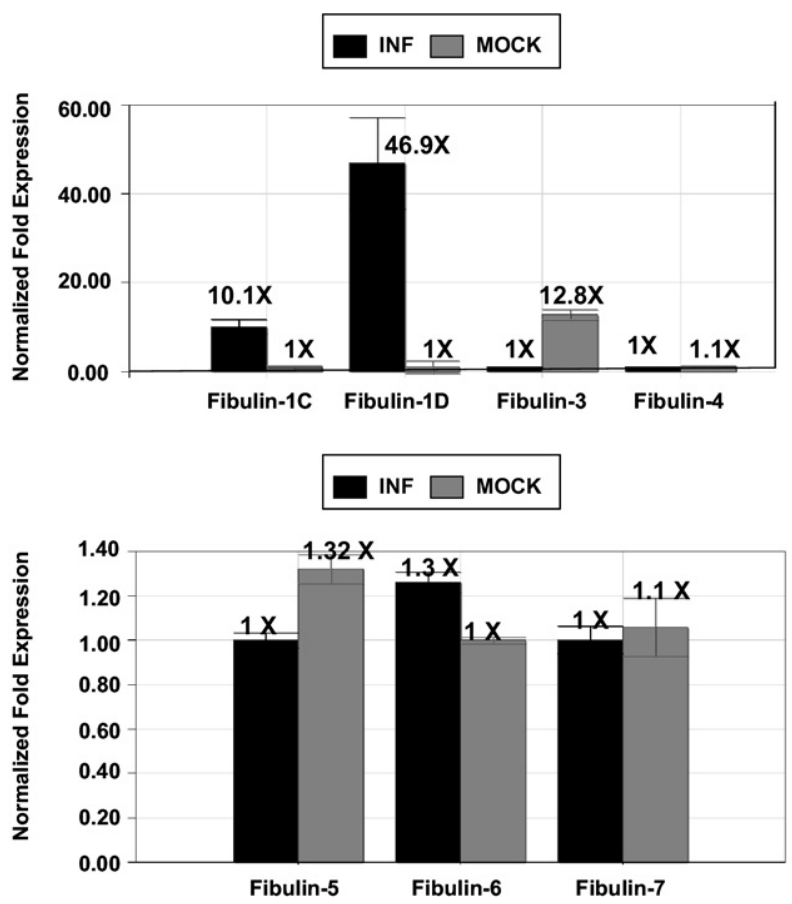

Figure 4. RT-PCR analysis of fibulins. Transcription analysis of fibulins in KSHV-infected DMVEC by RT-PCR. Shown are transcriptional analysis of fibulin family members fibulin-1C, fibulin-1D, fibulin-3, and fibulin-4 (A) as well as fibulin-5, fibulin-6, and fibulin-7 (B). Ten nanograms of cDNA from mock- and KSHV-infected DMVEC (10 days) were amplified by qRT-PCR using a group of fibulin gene-specific primers.

and 7 in infected versus mock-infected cells (Figure 4). We also found no demonstrable levels of either fibulin-1A or $-1 \mathrm{~B}$ in either control or infected DMVEC cells (data not shown). In contrast, fibulin-1C and -1D mRNA expression was upregulated 10 -fold and 47 -fold, respectively, 10 days after KSHV infection. Fibulins 3 and 5, like fibulin-2, were downregulated in KSHV-infected DMVEC. The fibulin-2, -3, and -5 data correlates with previous reports of their down-regulation in several human tumors. ${ }^{27}$ We observed no significant change in mRNA expression of fibulin- 6 and fibulin-7. Because there are no known associations of these two fibulins with cancer, they were excluded from further study (Figure 4). Thus, mRNA levels for fibulins 2, 3, and 5 were all reduced at 10 days after $\mathrm{KSHV}$ infection in DMVEC cells, whereas fibulins $1 \mathrm{C}(10 \times)$ and particularly $1 \mathrm{D}(47 \times)$ were significantly up-regulated (Figure 4).

\section{Down-Regulation of Fibulin-2, Fibulin-3, and Fibulin-5 in KSHV-Infected DMVEC Correlates with Increases in Viral LANA Transcription}

We examined the temporal expression of fibulin-1C, -1D, -2 , -3 , and fibulin-5 in KSHV-infected DMVEC by time course transcriptional analysis using quantitative realtime RT-PCR. For fibulin-1C, at 2 days after infection, we observed a marginal down-regulation of mRNA expression in KSHV-infected cells. However, at 5, 7, and 10 days after infection, we observed a consistent increase in
fibulin-1C and -1D mRNA expression in KSHV-infected DMVEC (Figure 5, A and B). There is a marginal, but insignificant, increase at 2 days for fibulin-1D. For fibulin-2 at 2 days after infection, we observed a marginal, but insignificant, up-regulation of mRNA expression followed by a consistent decrease in mRNA synthesis at 5, 7 , and 10 days after KSHV infection compared to controls (Figure 5C). We observed a similar pattern of mRNA expression for fibulin-3 as we found with fibulin-2 (Figure 5D). In contrast, with fibulin-5, we observed a temporary, but significant, down-regulation in mRNA synthesis at 5 and 7 days after KSHV infection compared to mock-infected control cells. In contrast, the difference in expression between infected and mock cells at day 10 was insignificant in this series of studies (Figure 5E). Taken together, fibulin-3 showed the greatest level of down-regulation followed by fibulins 5 and 2. The greatest level of transcriptional upregulation was observed for fibulin-1D (46.9-fold) followed by fibulin-1C (10.1-fold) (Figure 4A). Over the same time frame, we observed a consistent increase in LANA transcription in KSHV-infected cells compared to mock-infected control cells (Figure 5F). This suggests that virus-induced changes in these fibulins correlated with the time course of latent viral gene expression.

\section{Fibulin Recombinant Protein Suppression of KSHV-Induced VEGF}

Confluent KSHV-infected DMVEC cells were exposed for 48 hours to recombinant fibulin-3 or fibulin-5 and compared to KSHV-infected DMVEC alone or uninfected cells, to determine the ability of these fibulins to alter the levels of angiogenic cytokines that may have been elicited by KSHV (recombinant fibulin-2 is not commercially available). Using a human angiogenic cytokine ELISA screening assay, we observed that KSHV alone increased tumor necrosis factor (TNF), VEGF, IL-6, and basic fibroblast growth factor (FGF $\beta$ ) supernatant concentrations (Figure 6A). Recombinant fibulin-3 added to KSHV-infected cells reduced TNF and VEGF values to those seen in controls, but had no or little effect on FGFb. Recombinant fibulin-5 reduced VEGF values to those seen in controls, but elevated both TNF and FGF $\beta$ levels beyond those seen with KSHV infection alone. IL-6 results were particularly interesting. KHSV infection produced a four- to fivefold increase in IL-6 that was further enhanced by recombinant fibulin-3 addition, and was more than doubled by recombinant fibulin-5. Values between all four comparative groups were similar for IGF and transforming growth factor (TGF $\beta$ ) concentrations.

\section{Transcriptional Down-Regulation of VEGF by Fibulin-3 and Fibulin-5 Recombinant Proteins}

Transcriptional expression of KSHV LANA was demonstrated in all three KSHV-infected DMVEC cell preparations, and no LANA transcription was observed in mockinfected cells (Figure 6B). Transcriptional expression of VEGF subtypes (VEGF ${ }^{121}$, VEGF ${ }^{165}$, VEGF ${ }^{189}$ ) were observed to be down-regulated approximately three- and 
A Temporal Fibulin-1C Expression by qRT-PCR in KSHV Infected DMVEC cells

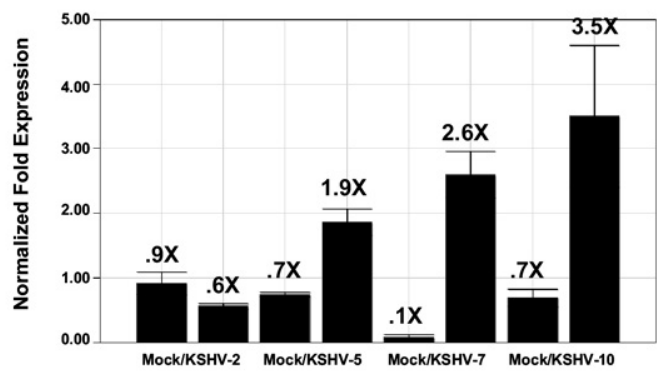

C in KSHV Infected DMVEC cells

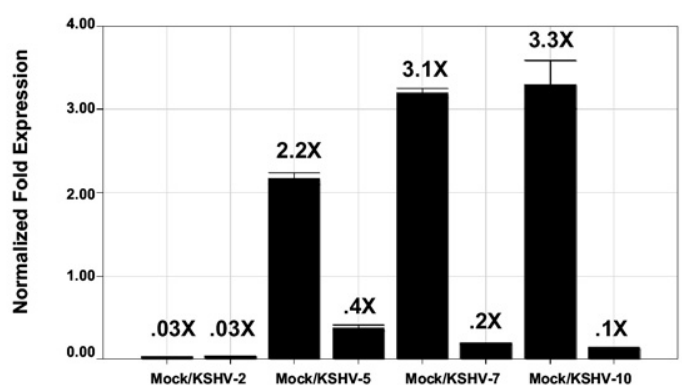
E in KSHV Infected DMVEC cells

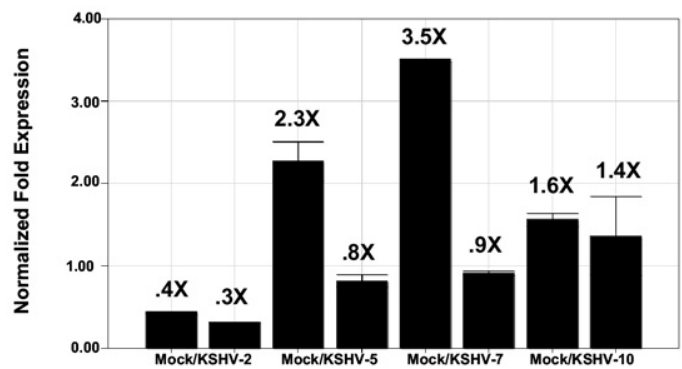

B Temporal Fibulin-1D Expression by qRT-PCR in KSHV Infected DMVEC cells

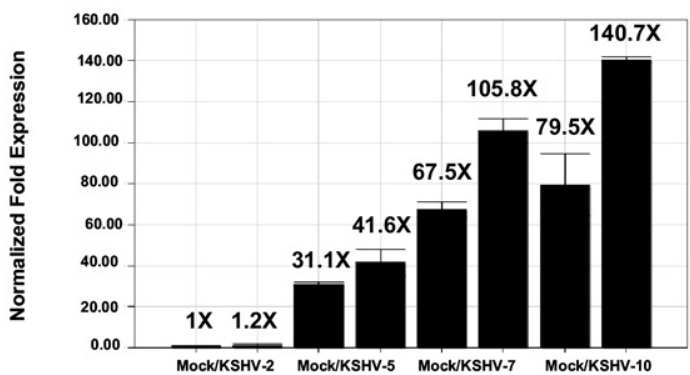

D Temporal Fibulin-3 Expression by qRT-PCR in KSHV Infected DMVEC cells

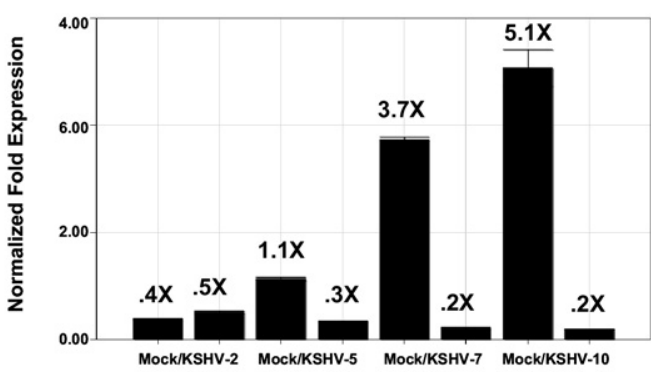

$\mathrm{F}$

Temporal LANA Expression by qRT-PCR in KSHV Infected DMVEC cells

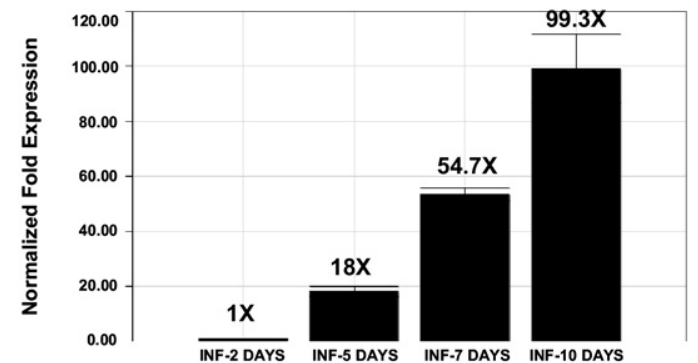

Figure 5. Temporal expression ( 2 to 10 days) of fibulins IC, ID, 2, 3, and 5 and LANA in KSHV-infected DMVEC determined by RT-PCR. A: Expression of fibulin-1C in KSHV- and mock-infected DMVEC cells after 2, 5, 7, and 10 days after infection. B: Temporal expression of fibulin-1D, mock, or KSHV infection. C: Temporal expression of fibulin 2, mock versus KSHV infection. D: Temporal expression of fibulin-3, mock versus KSHV infection. E: Temporal expression of fibulin-3, mock versus KSHV infection. F: Shows temporal expression of KSHV LANA in KSHV-infected DMVEC after 2, 5, 7, and 10 days after infection. Relative fold expression was normalized to GAPDH.

twofold in KSHV-infected DMVEC exposed to fibulin-3 or fibulin-5 recombinant proteins, respectively (Figure 6C).

\section{Fibulin-3 Recombinant Protein Suppression of KSHV Induction of VEGFR3}

Zhang et al reported that KSHV infection of endothelial cells leads to the activation of VEGFR3, which alters endothelial cell function and enhances virus infectivity. ${ }^{28}$ Therefore, after observing a significant reduction of VEGF protein elicited in supernatants of KSHV-infected DMVEC cells, as well as reduced transcriptional expression of VEGF mRNA in infected cells exposed to fibulin-3 and fibulin-5 recombinant proteins, we wanted to determine the effect of these proteins on the expression of VEGF receptors in infected cells. Using qRT-PCR, we observed a reduced transcriptional expression of VEGFR3 in
KSHV-infected DMVEC exposed for 48 hours to fibulin-3 recombinant protein with no significant change in the expression of VEGFR1 and VEGFR2 compared to KSHVinfected DMVEC in medium only (Figure 7, A, B, and C). In infected cells exposed to fibulin-5 protein, we observed a significant increase in expression of VEGFR1 with no significant change in expression of VEGFR2 and VEGFR3. We also observed a consistent induction of all three VEGF receptors in KSHV-infected DMVEC cells in comparison to mock-infected controls cells (Figure 7, A, B, and C).

\section{Expression of Fibulin-2 in PEL Cell Line}

We then turned to PEL cell lines derived from these B-cell lymphomas. We wanted to determine whether there was an inverse relationship between the basal levels of fibu- 
A Angiogenesis Cytokine Assay

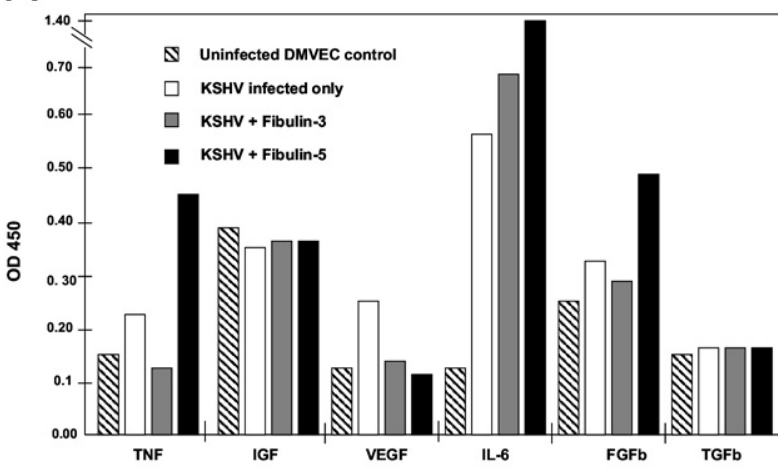

B

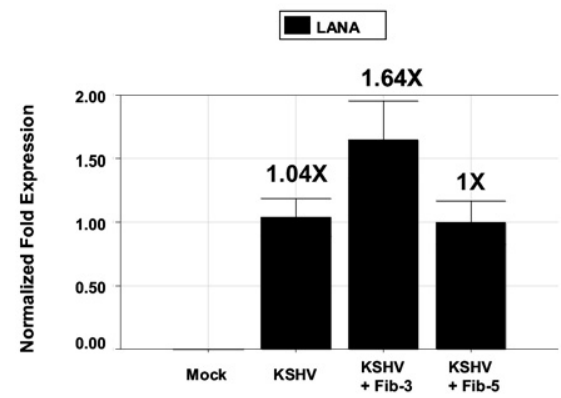

C VEGF after Exposure to Fibulin Protein

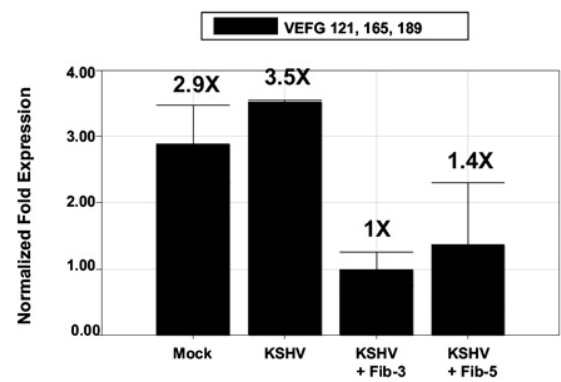

Figure 6. Analysis of angiogenic cytokines after KSHV infection and exposure to fibulins. A: A human angiogenic cytokine ELISA assay was performed with confluent 10-day normal and KSHV-infected cells. Results show the relative amounts of angiogenic cytokines TNF $\alpha$, IGF, VEGF, IL-6, FGFb, and $\mathrm{TGFb}$ in cellular supernatants elicited 48 hours after exposure to fibulin-3 and fibulin- 5 recombinant proteins compared to normal DMVEC cultures and KSHV alone. KSHV-infected DMVEC were exposed to $10 \mu \mathrm{g} / \mathrm{mL}$ of purified recombinant fibulin-3 protein or $10 \mu \mathrm{g} / \mathrm{mL}$ of purified recombinant fibulin- 5 protein. B: Shows real-time PCR analysis using RNA extracted from cell pellets for KSHV LANA at 48 hours after exposure to fibulin-3 or fibulin- 5 recombinant proteins compared to mock-treated and KSHV-treated cells alone. C: Shows real-time PCR analysis of KSHV-infected DMVEC exposed to fibulin-3 or fibulin-5 proteins for VEGF 121, 165, 189, at 48 hours after exposure to these recombinant proteins compared to mock or KSHV alone. Relative fold expression was normalized to GAPDH.

lin-2 in established PEL cell lines and the levels of KSHV replication in those lines.

The levels of KSHV replication in several PEL cell lines (JSC-1, BC3, BC2, BCBL-1, and HBL6) were determined by Southern blot analysis, using $10 \mu \mathrm{g}$ of total DNA/lane extracted from cell lysates without induction of the virus lytic cycle. We showed variable levels of KSHV genomic DNA replication in PEL cell lines when probed with ${ }^{32} \mathrm{P}$ labeled, 800-bp terminal repeat fragments from the KSHV genome (Figure 8A). This method measures terminal ends of viral genomes, indicating the degree of lytic viral replication occurring in the PEL cell line in the absence of TPA induction. The relative levels of viral expression found were consistent with those reported in the literature. ${ }^{29}$ The JSC- 1 cell line, established by Cannon et al, ${ }^{29}$ 2000, revealed the highest level of KSHV replication followed by BC3. Similar levels of viral DNA were found in BC2 and HBL6 PELs, but no detectable level of KSHV DNA was observed in the BCBL-1 cell line (Figure 8A). To determine the comparable expression levels of fibulin-2 in several of these PEL lines, we performed Western blot analysis on lysates from cultured primary effusion lymphoma cell lines JSC-1, BC3, BC2, and HBL6 (BJAB cells were used a control). None of these PEL lines had demonstrable fibulin-2 expression in sharp contrast to that observed in DMVEC cells (Figure 8B). We also assayed the PEL cell lines for expression of fibulin-5, and no expression was observed (data not shown). Fibulin-1C and fibulin-1D were not assayed because there are no commercial antibodies available for fibulin-1C and fibulin-1D. Moreover, the transformation of these B cells could negatively regulate fibulin- 2 expression because BJAB cells are also negative for fibulin-2 expression.

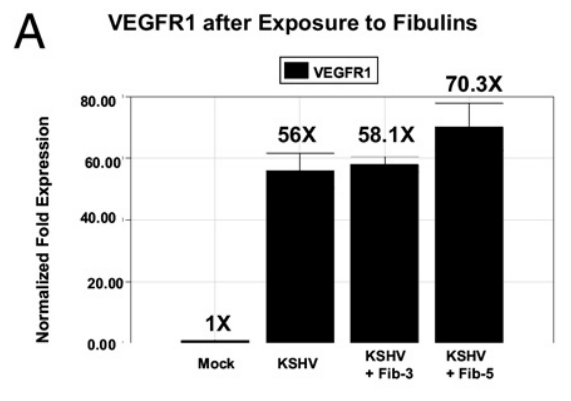

B VEGFR2 after Exposure to Fibulins

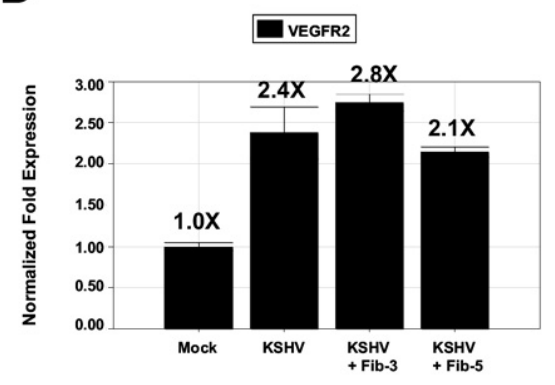

C VEGFR3 after Exposure to Fibulins

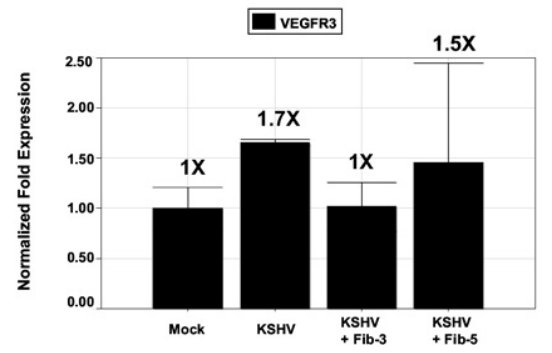

Figure 7. RT-PCR analysis of VEGF receptors. Real-time PCR analysis of KSHV-infected DMVEC exposed to fibulin-3 or fibulin- 5 proteins for VEGFR1 (A), VEGFR2 (B), and VEGFR3 (C) at 48 hours after exposure to recombinant fibulin -3 and fibulin -5 proteins compared to mock and KSHV alone infected. Relative fold expression was normalized to GAPDH. 

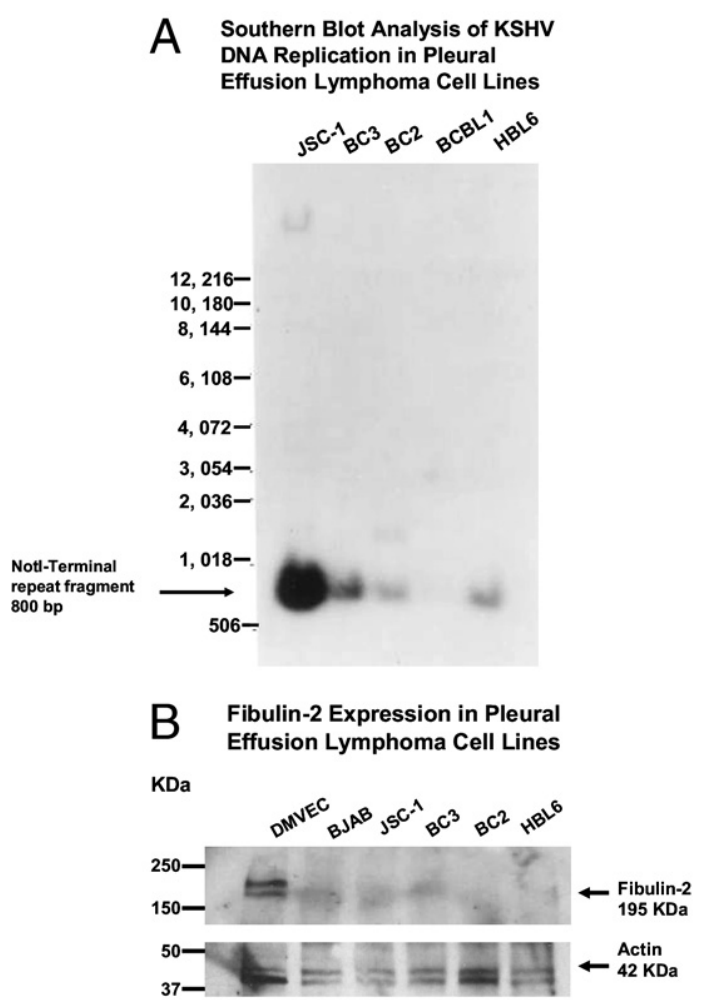

Figure 8. Southern blot screening of KSHV replication and fibulin-2 expression in PEL cell lines. A: DNA from PEL cell lines were screened by Southern blot hybridization using a KSHV terminal repeat probe that was ${ }^{32} \mathrm{P}$ labeled. Hybridized fragment sizes are given in base pairs for the respective lymphoma cell line. A 1-kb DNA ladder was used to determine fragment sizes. B: A Western blot performed with lysates from PEL cell lines probed with a rabbit polyclonal antibody to fibulin-2, compared to DMVEC and a B-cell control, BJAB. Actin was used as a loading control, and protein size makers are given in kilodaltons.

\section{Variable Levels of Viral Burden in KS Tissue}

To our knowledge there are no reports of fibulin-2 expression analyses in KS tissue or any reports of fibulin-2 involvement in the pathogenesis of KS. Therefore we first examined archival KS tissue from a lesion that represented the most advanced stage of KS disease, marked by increased levels of virus replication and increased numbers of spindle cells, resembling the nodular form of $\mathrm{KS}$. As shown in Figure 9A-9D, after IHC staining for KSHV LANA, these lesions express variable levels of KSHV infection that suggest a correlation between viral burden and stage of KS disease. Figure 9A and 9B represents low levels of viral burden with little to no spindle cells and Figures 9C and 9D represent intermediate to heavy viral burden with a high number of spindle cells respectively.

\section{Fibulin-2 Expression in Tissue Microarrays}

Tissue microarrays were initially stained for KSHV LANA by immunohistochemistry and analyzed by bright-field microscopy. A counterstain was not included to avoid discrepancies with substrate colors. Color development for LANA was performed with DAB, and LANA-positive cells were visualized by an intense brown nuclear punc- tuate staining pattern. Stained tissue cores were observed at $\times 200$ magnification. Stained tissue cores were divided into KSHV LANA-positive (tumor) and KSHV LANA-negative (nontumor) groups. There were 19 cores designated as being positive for KSHV LANA, and 67 designated as being negative after examination of 50 optical fields for each tissue core examined. Fibulin-2 was stained with DAB, and LANA was stained with the Vector Red substrate for alkaline phosphatase. LANApositive cells in the tumor were visualized by an intense red nuclear punctuate staining pattern (Figure 10). Fibulin-2-positive cells in the tumors were visualized by brown cytoplasmic staining pattern (Figure 10). Dualstained tissue cores were observed at $\times 200$ magnification. Although we were supplied with 126 tissue samples, we were only able to analyze 86 that survived intact through tissue processing. We identified 19 (22\%) LANApositive KS tumors and 67 (78\%) LANA-negative tissue samples. All 86 samples were found to be fibulin-2 positive, and 19 of 19 LANA-positive cores showed reduced expression of fibulin-2 in LANA-positive spindle cell regions (Figure 1, Table 1). If KSHV LANA-positive tumor core spindle cell regions in 50 optical fields exhibited a $>50 \%$ reduction in fibulin-2, they were designated reduced in expression for fibulin-2. The observation of 19 of $19(100 \%)$ KS tumors that were LANA-positive resulting in reduced expression of fibulin-2 is highly significant when compared to LANA-negative tumors. Of the 19 LANA-positive tumors, all 19 had reduced fibulin-2 levels compared to 0 of 67 LANA-negative tumor specimens, which all had high levels of fibulin-2 expression, especially in non-spindle cell regions.

\section{IHC and Kaposi's Sarcoma Tissue Microarrays}
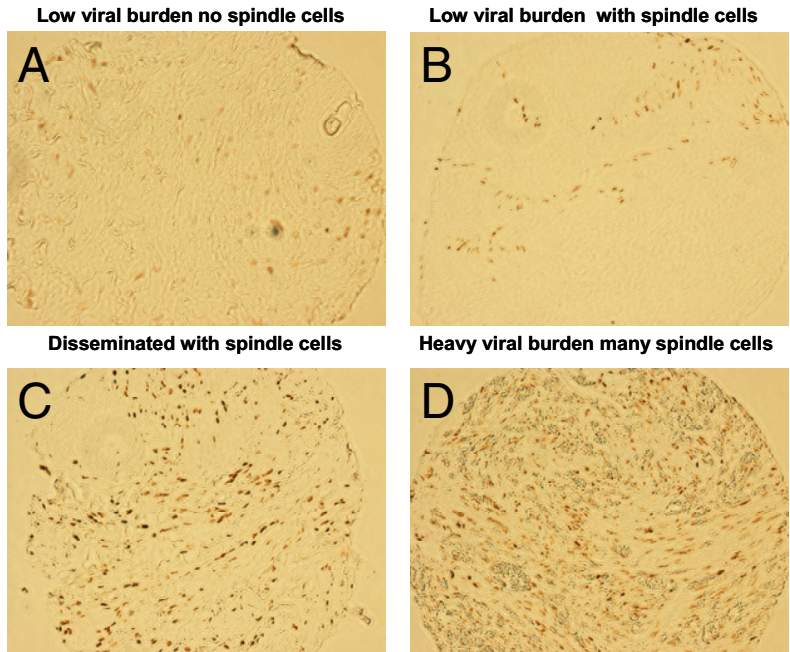

Figure 9. Immunohistochemical analysis of a human KS tissue array, stained for KSHV LANA, and analyzed by IHC. Illustrations represent tumor tissue with different viral burdens and different number of spindle cells. A: Tumor tissue from a patient with low viral burden with little or no spindle cells; $\mathbf{B}$ : Tumor tissue with some spindle cells; C: Disseminated KSHV infection with many spindle cells; D: Heavy viral burden with a high number of spindle cells. 
Dual labeled IHC for fibulin-2 and KSHV LANA

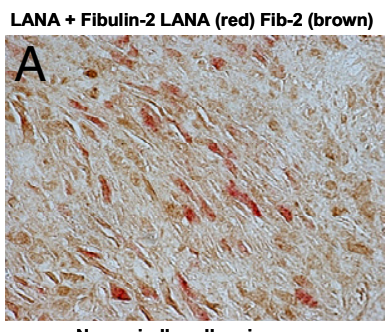

Non-spindle cell region
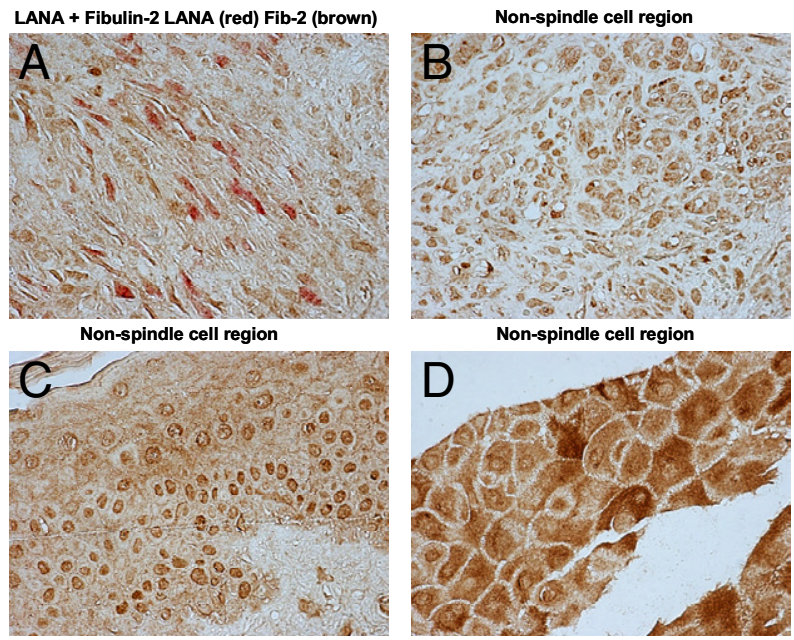

Figure 10. Fibulin-2 expression in human KS tumor tissue. Briefly, paraffinembedded KS tissue was deparaffinized in xylene and hydrated in graded alcohol, and antigen unmasking was performed in citrate buffer in a microwave. Cells were stained by dual-labeled IHC for KSHV LANA (Vector Red, red) and fibulin-2 (DAB brown). No counterstain was used in this procedure. Bright-field images were photographed on a Nikon TE2000S microscope at total magnification of $\times 200$. A: A spindle cell region; $\mathbf{B}-\mathbf{D}$ : non-spindle cell regions observed in a representative KS tissue specimen.

\section{Discussion}

In this study, we showed the characteristic morphological changes observed in DMVEC cells after KSHV infection in vitro with the similar changes seen in KS tumor tissue (Figure 1). This is the first report indicating down-regulation of fibulin-2 protein and message in KSHV-infected DMVEC cells, which are primary cells that mimic the histopathology of Kaposi's sarcoma in vivo, and in human $\mathrm{KS}$ tumor tissue compared to nontumor tissue in the same mix of samples. We also report here the temporal transcriptional down-regulation of fibulin-3 and fibulin-5 in KSHV-infected DMVEC cells. In contrast, we observed the temporal transcriptional up-regulation of fibulin-1C and $-1 \mathrm{D}$. We propose that the dysregulation of fibulin family members such as fibulin-2, $-3,-5,1 \mathrm{C}$, and $1 \mathrm{D}$ likely contribute to KSHV-induced pathogenesis in KS. Dysregulation of multiple members of the fibulin family of extracellular matrix proteins in KS is a novel observation with implications for viral-induced changes in endothelial cell proliferation, migration, angiogenesis, and invasion that could directly contribute to KS tumorigenesis. We propose that down-regulation or loss of fibulin-2 compromises the structural integrity of the basement membrane due to loss of interactions between fibulin-2 and several ECM proteins. ${ }^{7}$
In addition, this is the first report of transcriptional down-regulation of fibulin-3 and fibulin- 5 and transcriptional up-regulation of fibulin-1C and -1D in KSHV-infected DMVEC. Other fibulin isoforms (fibulin 1A, 1B) were not expressed at detectable levels in our transcription assay. This finding is consistent with results from other laboratories that report fibulin-1C and -1D are the predominantly expressed fibulin-1 spliced variants in various cell types and, further, that other fibulin- 1 isoforms are not expressed or poorly expressed in these same cells. ${ }^{30}$ Different roles have been suggested for fibulin-1C and -1D based on their binding to the basement membrane protein nidogen. ${ }^{31}$ Fibulin-1C binds to nidogen with a 30-fold higher affinity than does fibulin-1D. More recent studies have shown increased fibulin-1C:1D mRNA ratios in ovarian carcinomas. ${ }^{14}$ Fibulin $1 \mathrm{C}$ mRNA expression can be induced by estrogen in estrogen receptor $\alpha$-positive ovarian tumor cell lines, ${ }^{14,32}$ and overexpression of fibulin-1C mRNA in ovarian cancer has been associated with tumor progression. ${ }^{14}$

Fibulin-5 is thought to have an important role in endothelial cell function, vessel repair, and angiogenesis. We observed early down-regulation of fibulin-5 message in KSHV-infected DMVEC, which returned to mock control cell values by day 10 . This finding is also intriguing because fibulin-5 mediates endothelial cell adhesion through its integrin-binding RGD domain that interacts with the angiogenic-associated integrin alphavbeta3. ${ }^{33}$ $\mathrm{KSHV}$ is also known to stimulate expression of the proangiogenic cytokine VEGF, a potent inhibitor of fibulin-5 expression. ${ }^{20}$ This would suggest that fibulin-5 downregulation in infected DMVEC could be mediated by an increase in expression of VEGF. c-Myc has also been found to repress fibulin-5 expression. Lui et al, 2007, reported that KSHV LANA, which is an abundant latent protein expressed in KSHV-infected PEL cell lines, stabilizes and activates c-Myc. ${ }^{34}$ This suggests that activation of c-Myc may also enhance suppression of fibulin-5 in KSHV-infected cells.

TGF- $\beta$ is known to stimulate fibulin- 5 expression in endothelial cells, ${ }^{20}$ and recently, Di Bartolo et al, 2008, showed that KSHV LANA inhibits TGF- $\beta$ signaling through epigenetic silencing of the TGF- $\beta$ type II receptor. ${ }^{35}$ They demonstrated that this pathway is blocked in PEL cells via downregulation of the TGF- $\beta$ type $\|$ receptor. $^{35}$ They also concluded that KSHV LANA associates with the TGF- $\beta$ promoter leading to methylation and deacetylation of proximal histones. Xie et al, 2008, reported that basement membrane-derived fibulins 1 and 5 function as angiogenic inhibitors that suppress tumor growth. ${ }^{36}$

Table 1. Analysis of KS Tissue Microarrays

\begin{tabular}{cccccc}
\hline $\begin{array}{c}\text { Tumor cores } \\
\text { spotted on } \\
\text { TMA }\end{array}$ & $\begin{array}{c}\text { Intact tumor } \\
\text { cores }\end{array}$ & $\begin{array}{c}\text { Total LANA-positive } \\
\text { cores }\end{array}$ & $\begin{array}{c}\text { LANA-negative } \\
\text { tumor cores }\end{array}$ & $\begin{array}{c}\text { Fibulin-2-positive } \\
\text { cores }\end{array}$ & $\begin{array}{c}\text { Tumor cores showing } \\
\text { reduced expression } \\
\text { of fibulin-2 in LANA } \\
\text { spindle cell regions }\end{array}$ \\
\hline 126 & 86 & 19 & 67 & 126 & $19 / 19$ \\
$100 \%$ & $68 \%$ & $22 \%$ & $78 \%$ & All examined & $100 \%$ \\
\hline
\end{tabular}

Intact tumor cores are tumor cores examined that were not destroyed by processing during mounting fixation or staining. 
Temporal expression of fibulin-2, -3 , and -5 was largely down-regulated 5,7 , and 10 days after KSHV infection that correlates with time periods in which there was a consistent increase in KSHV LANA transcriptional expression. VEGF protein expression and transcription induced by KSHV was inhibited by addition of recombinant fibulin-3 or fibulin-5 for 48 hours. The fact that IL-6 and $\mathrm{TNF} \alpha$ were found to be up-regulated in this assay by fibulin- 5 suggests that fibulin- 5 may have a role in proliferative responses during $\mathrm{KSHV}$ infection.

VEGFR3 activation and expression induced by KSHV glycoprotein B $(\mathrm{gB})$ has been shown to enhance KSHV infectivity and play an important role in KSHV-mediated transformation. ${ }^{28}$ Down-regulation of VEGFR3 after exposure to fibulin-3 recombinant protein was found to be marginal. This level of suppression was obtained with only $10 \mu \mathrm{g} / \mathrm{mL}$ of purified protein and may require higher levels of fibulin-3 protein that could only be obtained by vector delivery of fibulin-3 to cells or tissue.

We observed no detectable expression of fibulin-2 or fibulin-5 in any of the PEL lines tested regardless of the degree of KSHV lytic replication observed. The absence of demonstrable fibulin-2 or fibulin-5 expression in PELs that express varying levels KSHV replication may suggest low to no demonstrable expression of fibulin-2 or fibulin-5 in PEL cell lines in general, and/or that the presence of KHSV replication reduces endogenous fibulin-2 and fibulin-5 expression to undetectable amounts, independent of KSHV replication levels.

Tissue microarrays revealed that LANA-positive KS tumors showed a reduction in fibulin-2 expression when compared to LANA-negative tissue. Among the 19 LANApositive tumors observed, all 19 showed reduced expression of fibulin-2 in LANA-positive spindle cell regions. This is a significant finding, and we realize that examination of more tumor specimens is required. We intend to examine tissue fibulin-2 levels directly by real-time PCR in the future after acquiring additional KS tumors along with negative matched control specimens.

The dysregulation of the fibulin-2 extracellular matrix protein binding partners fibronectin and tropoelastin suggest that KSHV and/or down-regulation of fibulin-2 can affect the transcription of these essential ECM binding proteins. Tropoelastin has also been shown to interact with fibulin-5, and this interaction is likely involved in the initial steps of elastic fiber assembly. ${ }^{37}$

Fibronectin is essential for many cellular functions including migration, proliferation, and differentiation. ${ }^{38}$ Down-regulation of fibronectin transcription has also been shown to be associated with a highly metastatic form of murine mammary adenocarcinoma cells. ${ }^{39}$ These authors demonstrated that loss of fibronectin expression in these cells is not due to changes in the fibronectin promoter sequence but is the result of negative transcriptional regulation. ${ }^{39}$ More recent studies using cDNA arrays to identify genes associated with the more metastatic variant of $\mathrm{MM} 3$ adenocarcinoma cells as compared to the parent tumor (M3) found that fibronectin was one of only two genes significantly down-regulated among the genes analyzed in the study. ${ }^{40}$ They suggested that fibronectin may behave as an important metastasis sup- pressor protein in mammary cancer. ${ }^{40}$ In addition, viral proteins have been known to repress fibronectin transcription. ${ }^{41}$ As an example, the oncogenic adenovirus E1a protein has been shown to suppress fibronectin transcription in $3 Y 1$ rat fibroblasts. ${ }^{41}$ Finally, a study by Torre et al revealed significantly reduced serum levels of fibronectin in HIV-infected patients with $\mathrm{KS} .{ }^{42}$

Tropoelastin is a soluble precursor of elastin that can be induced by UV irradiation and degraded by metalloproteinases such as macrophage elastase (MMP12 ${ }^{43}$ ). Loss of tropoelastin can lead to developmental tissue disorders such as aneurysms, atherosclerosis, and loss of skin elasticity. Tropoelastin along with fibronectin are essential ECM proteins critical for wound healing.

Finally, we report here the transcription of fibulin-3, -2 , and -5 are down-regulated in DMVEC cells infected with KSHV. The dysregulation of these additional fibulin family members other than fibulin-2 (fibulin-1C, -1D, -3, and -5) after KSHV infection of DMVEC suggests that virus targets fibulins to compromise the integrity of the extracellular matrix thereby altering cell proliferation, migration, invasion, and angiogenesis. This suggests that fibulins can interact directly with viral gene products involved in transformation and may serve as potential targets for a novel treatment strategy for KS disease. The results of this study suggest that the dysregulation of fibulin-2 and its ECM binding partners likely contributes to KS pathogenesis. Understanding the underlying mechanisms involved in fibulin-mediated contributions to KS pathogenesis will require further studies.

\section{Acknowledgments}

We thank Drs James E.K. Hildreth and Diana Marver for their advice in the preparation of this manuscript. We also thank Jared Elzey for editing this manuscript.

\section{References}

1. Kedes DH, Operskalski E, Busch M, Kohn R, Flood J, Ganem D: The seroepidemiology of human herpesvirus 8 (Kaposi's sarcoma-associated herpesvirus): distribution of infection in KS risk groups and evidence for sexual transmission. Nat Med 1996, 8:918-924

2. Schultz TF: Kaposis's sarcoma-associated herpesvirus (human herpesvirus 8): epidemiology and pathogenesis. J Antimicrobial Chemother 2000, 45:15-27

3. Sitas F, Newton R: Kaposi's sarcoma in South Africa. J Natl Cancer Inst Monogr 2001, 28:1-4

4. Chang Y, Cesarman E, Pessin MS, Lee F, Culpepper J, Knowles DM, Moore PS: Identification of herpesvirus-like DNA sequences in AIDSassociated Kaposi's sarcoma. Science 1994, 266:1865-1869

5. Hayward GS, Alcendor DJ, Boger RA: The role of KSHV in the pathogenesis of Kaposi's sarcoma. In Viral Oncology: Basic Science and Clinical Applications. Edited by Khalili K, Jeang KT. Hoboken, NJ, Wiley-Blackwell, 2010, pp 377-407

6. Simonart T, Hermans P, Schandene L, Van Vooren JP: Phenotypic characteristics of Kaposi's sarcoma tumour cells derived from patch-, plaque- and nodular-stage lesions: analysis of cell cultures isolated from AIDS and non-AIDS patients and review of the literature. $\mathrm{Br} J$ Dermatol 2000, 143:557-563

7. Timpl R, Sasaki T, Kostka G, Chu ML: Fibulins: a versatile family of extracellular matrix proteins. Nat Rev Mol Cell Biol 2003, 4:479-489

8. Argraves WS, Greene LM, Cooley MA, Gallagher WM: Fibulins: physiological and disease perspectives. EMBO Rep 2003, 12:1127-1131 
9. Pan TC, Kostka G, Zhang RZ, Timpl R, Chu ML: Complete exon-intron organization of the mouse fibulin-1 gene and its comparison with the human fibulin-1 gene. FEBS Lett 1999, 444:38-42

10. Pan TC, Sasaki T, Zhang RZ, Fässler R, Timpl R, Chu ML: Structure and expression of fibulin-2, a novel extracellular matrix protein with multiple EGF-like repeats and consensus motifs for calcium binding J Cell Biol 1993, 123:1269-1277

11. Kobayashi N, Kostka G, Garbe JH, Keene DR, Bächinger HP Hanisch FG, Markova D, Tsuda T, Timpl R, Chu ML, Sasaki T: A comparative analysis of the fibulin protein family, biochemical characterization, binding interactions and tissue localization. J Biol Chem 2007, 282:11805-11816

12. Fisher SA, Rivera A, Fritsche LG, Keilhauer CN, Lichtner P, Meitinger $\mathrm{T}$, Rudolph $\mathrm{G}$, Weber $\mathrm{BH}$ : Case-control genetic association study of fibulin-6 (FBLN6 or HMCN1) variants in age-related macular degeneration (AMD). Hum Mutat 2007, 4:406-413

13. de Vega S, Iwamoto T, Nakamura T, Hozumi K, McKnight DA, Fisher LW, Fukumoto S, Yamada Y: TM14 is a new member of the fibulin family (fibulin-7) that interacts with extracellular matrix molecules and is active for cell binding. J Biol Chem 2007, 282:30878-30888

14. Moll F, Katsaros D, Lazennee G, Hellio N, Giacalone PL, Chalbos D, Maudelonde T, Rochefort H, Pujol P: Estrogen induction and overexpression of fibulin-1C mRNA in ovarian cancer cells. Oncogene 2002 , 7:1097-1107

15. Greene LM, Twal WO, Duffy MJ, McDermott EW, Hill AD, O'Higgins NJ, McCann AH, Dervan PA, Argraves WS, Gallagher WM: Elevated expression and altered processing of fibulin-1 protein in human breast cancer. Br J Cancer 2003, 6:871-878

16. Yi $\mathrm{CH}$, Smith DJ, West WW, Hollingsworth MA: Loss of fibulin-2 expression is associated with breast cancer progression. Am J Pathol 2007, 5:1535-1545

17. Albig AR, Niel JR, Schiemann WP: Fibulins 3 and 5 antagonize tumor angiogenesis in vivo. Cancer Res 2006, 5:2621-2629

18. Gallagher WM, Greene LM, Ryan MP, Sierra V, Berger A, LaurentPuig P, Conseiller E: Human fibulin-4: analysis of its biosynthetic processing and mRNA expression in normal and tumor tissue. FEBS Lett 2001, 1:59-66

19. Schiemann WP, Blobe GC, Kalume DE, Pandey A, Lodish HF: Context-specific effects of fibulin-5 (DANCE/EVEC) on cell proliferation, motility, and invasion. Fibulin-5 is induced by transforming growth factor-beta and affects protein kinase cascades. J Biol Chem 2002, 30:27367-27377

20. Albig AR, Schiemann WP: Fibulin-5 antagonizes vascular endothelial growth factor (VEGF) signaling and angiogenic sprouting by endothelial cells. DNA Cell Biol 2004, 23:367-379

21. Kobayashi N, Kostka G, Garbe JH, Keene DR, Bachinger HP, Hanisch FG, Markova D, Tsuda T, Timpl R, Chu ML, Sasaki T: A comparative analysis of the fibulin protein family. Biochemical characterization, binding interactions, and tissue localization. J Biol Chem 2007, 282:11805-11816

22. Reinhardt DP, Sasaki T, Dzamba BJ, Keene DR, Chu ML, Gohring W, Timpl R, Sakai LY: Fibrillin1 and fibulin-2 interact and are colocalized in some tissues. J Biol Chem 1996, 271:19489-19496

23. Olin Al, Morgelin M, Sasaki T, Timpl R, Heinegard D, Aspberg A: The proteoglycans aggrecan and versican form networks with fibulin-2 through their lectin domain binding. J Biol Chem 2001, 276:1253-1261

24. Utani A, Nomizu, M, Yamada Y: Fibulin-2 binds to the short arms of laminin-5 and laminin-1 via conserved amino acid sequences. J Biol Chem 1997, 272:2814-2820

25. Sasaki T, Gohring W, Pan TC, Chu ML, Timpl R: Binding of mouse and human fibulin-2 to extracellular matrix ligands. J Mol Biol 1995, 254:892-899

26. Hopf M, Gohring W, Kohfeldt E, Yamada Y, Timpl R: Recombinant domain IV of perlecan binds to nidogens, laminin-nidogen complex fibronectin, fibulin-2 and heparin. Eur J Biochem 1999, 259:917-925
27. Gallagher WM, Currid CA, Whelan LC: Fibulins and cancer: friend or foe? Trends Mol Med 2005, 11:336-340

28. Zhang X, Wang JF, Chandran B, Persaud K, Pytowski B, Fingeroth J, Groupman JE: Kaposi's sarcoma-asoociated herpesvirus activation of vascular endothelial growth factor receptor 3 alters endothelial function and enhances infection. J Biol Chem 2005, 28:26216-26224

29. Cannon JS, Ciufo D, Hawkins AL, Griffin CA, Borowitz MJ, Hayward GS, Ambinder RF: A new primary effusion lymphoma-derived cell line yields a highly infectious Kaposi's sarcoma herepesvirus-containing supernatant. J Virol 2000, 21:10187-10193

30. Tran H, Mattei M, Godyna S, Argraves WS: Human fibulin-1D: molecular cloning, expression and similarity with S1-5 protein, a new member of the fibulin gene family. Matrix Biol 1997, 15:479-493

31. Sasaki T, Gohring W, Pan TC, Chu ML, Timpl R: Binding of mouse and human fibulin-2 to extracellular matrix ligands. J Mol Biol 1995 5:892-899

32. Bardin A, Moll F, Margueron R, Delfour C, Chu ML, Maudelonde T, Cavailles V, Pujol P: Transcriptional and post-transcriptional regulation of fibulin-1 by estrogen leads to differential induction of messenger ribonucleic acid variants in ovarian and breast cancer. Endocrinology 2005, 2:760-768

33. Nakamura T, Ruiz-Lozano $P$, Lindner $V$, Yabe D, Taniwaki M, Furukawa Y, Kobuke K, Tashiro K, Lu Z, Andon NL, Schaub R, Matsumori A, Sasayama S, Chien KR, Honjo T: DANCE, a novel secreted RGD protein expressed in developing, atherosclerotic, and balloon-injured arteries. J Biol Chem 1999, 274:22476-22483

34. Liu J, Martin HJ, Liao G, Hayward SD: The Kaposi's sarcoma-associated herpesvirus LANA protein stabilizes and activates c-Myc. J Virol 2007, 19:10451-10459

35. Di Bartolo DL, Cannon M, Liu YF, Renne R, Chadburn A, Boshoff C, Cesarman E: KSHV LANA inhibits TGF-beta signaling through epigenetic silencing of the TGF-beta type II receptor. Blood 2008, 9:47314740

36. Xie L, Palmsten K, MacDonald B, Kieran MW, Potenta S, Vong S, Kalluri R: Basement membrane derived fibulin-1 and fibulin-5 function as angiogenesis inhibitors and suppress tumor growth. Exp Biol Med (Maywood) 2008, 2:155-162

37. Nonaka R, Onoue S, Wachi H, Sata F, Urban Z, Starcher BC, Seyama $Y$ : DANCE/fibulin-5 promotes elastic fiber formation in tropoelastin isoform-dependent manner. Clin Biochem 2009, 7-8:713-721

38. Brenner KA, Corbett SA, Schwarzbauer JE: Regulation of fibronectin matrix assembly by activated Ras in transformed cells. Oncogene 2000, 19:3156-3163

39. Webajh SE, Urtreger AJ, Puricelli LI, de Lustig ES, Joffe EBK, Kornblitt AR: Downregulation of fibronectin transcription in highly metastatic adenocarcinoma cells. FEBS Letters 1998, 440:277-281

40. Urtreger AJ, Werbajh SE, Verrecchia F, Mauviel A, Puricelli LI, Kornblihtt AR, Bal de Kier Joffe ED: Fibronectin is directly downregulated in murine mammary adenocarcinoma cells with high metatstaic potential. Oncol Rep 2006, 6:1403-1410

41. Nakamura T, Nakamura T, Tsunoda S, Nakada S, Oda K, Tsuri $H$, Wada A: Induction of E1A-responsive negative factors for transcription of the fibronectin gene in adenovirus E1-transformed rat cells. J Virol 1992, 11:6436-6450

42. Torre D, Zeroli C, Martegani R, Pugliese A, Basilico C, Speranza F: Levels of the bcl-2 protein, fibronectin and alpha (5) beta (1) fibronectin receptor in HIV-1-infected patients with Kaposi's sarcoma. Microbes Infect 2000, 15:1831-1843

43. Seo JY, Lee SH, Youn CS, Choi HR, Rhie G, Cho KH, Kim KH, Park $\mathrm{KC}$, Eun $\mathrm{HC}$ Chung $\mathrm{JH}$ : Ultraviolet radiation increases tropoelastin mRNA expression in the epidermis of human skin in vivo. J Invest Dermatol 2001, 6:915-919 